\title{
Procesos de feedback para fomentar la autorregulación con soporte tecnológico en la educación superior: Revisión sistemática
}

\author{
(Self-Regulated Feedback Processes Enhanced by \\ Technology in Higher Education: a Systematic Review)
}

\author{
Begoña Gros Salvat \\ Elena Cano García \\ Universidad de Barcelona (España)
}

DOI: $\underline{\text { https://doi.org/10.5944/ried.24.2.28886 }}$

\section{Cómo referenciar este artículo:}

Gros Salvat, B., y Cano García, E. (2021). Procesos de feedback para fomentar la autorregulación con soporte tecnológico en la educación superior: Revisión sistemática. RIED. Revista Iberoamericana de Educación a Distancia, 24(2), pp. 107-125. https://doi.org/10.5944/ried.24.2.28886

\section{Resumen}

El desarrollo del aprendizaje autorregulado en educación superior puede darse a través del fomento del juicio evaluativo, el cual, a su vez, puede adquirirse mediante procesos de evaluación entre iguales o de autoevaluación en los que se proporcione un feedback proporcionando andamiaje a los componentes cognitivos, metacognitivos y afectivos de la autorregulación. La tecnología puede suponer un valor añadido en dicho proceso. Sin embargo, la literatura parece apuntar usos de la tecnología más instrumentales que favorecedores de la autorregulación. En el presente artículo se presentan los resultados de un proceso de revisión sistemática sobre las aportaciones recientes que la bibliografía de referencia realiza en torno al rol de la tecnología para soportar el feedback. Los hallazgos muestran que, pese al aumento de aplicaciones y posibilidades técnicas que ofrecen las tecnologías digitales, el uso que se le da sigue estando vinculado a la gestión y almacenamiento de los datos, a la rapidez y facilidad de la calificación o, en algunos casos, al aumento de la motivación de los estudiantes. Sin embargo, los beneficios del uso de la tecnología en términos de los procesos de aprendizaje autorregulado que dichas tecnologías podrían reportar con un uso activo de los estudiantes siguen siendo todavía un campo pendiente para la investigación.

Palabras clave: aprendizaje autorregulado; educación superior; tecnologías de la información y de la comunicación; retroalimentación. 


\begin{abstract}
Self-regulated learning in higher education can be developed through the promotion of evaluative judgment which can be acquired through peer evaluation or self-evaluation processes. In these processes feedback must be provided for scaffolding the cognitive, metacognitive and affective components of self-regulation. Technology can add value to this process. However, the literature seems to state an instrumental use of technology more than a use helpful for self-regulated learning. This paper presents the results of a systematic review process on the recent contributions regarding the role of technology to support feedback. The findings show that, despite the increase in software and technical possibilities offered by new digital technologies, the use that is given to current digital technologies continues to be linked to the management and storage of data, the speed and ease of data processing. qualification or, in some cases, increasing student motivation. However, the benefits of the use of technology in terms of the self-regulated learning processes that such technologies could bring about with an active use of students is still a pending field for research.
\end{abstract}

Keywords: self-regulated learning; higher education; information and communication technologies; feedback.

La presencia de las tecnologías digitales en los procesos de enseñanza se ha venido incrementando en los últimos años (EACEA, 2019; EDUCAUSE 2020) y se ha extendido masivamente como consecuencia de la COVID-19 (UNESCO, 2020). Los beneficios del empleo de las tecnologías digitales en la enseñanza están profusamente documentados (Gros y Suárez, 2016). También ha sido sobradamente analizada la utilidad de la tecnología para la evaluación y el feedback (Rodríguez e Ibarra, 2011; Gikandi y Morrow, 2014; Barberà, 2016). Sin embargo, los modelos pedagógicos de evaluación y la concepción de feedback en la que se inscribe el uso de la tecnología son, muy usualmente, diseños tradicionales con escasa participación del estudiante. El cambio hacia un nuevo paradigma en el que el estudiante sea el protagonista y responsable de autorregular su aprendizaje tomando parte en los procesos de evaluación y feedback mediante el desarrollo del juicio evaluativo parece estar progresando. Desde esta perspectiva, la tecnología supera algunas funciones tradicionales (facilitar la gestión deinformación, automatizar procesos de calificación, acelerar la obtención de respuestas correctas o generar mensajes automáticos para proveer feedback) para pasar a tener otro papel. Su rol ha de ser el de dar soporte a la actividad del estudiante para representarse una tarea, comprender los criterios de evaluación (y participar en el establecimiento de los mismos), monitorear su propio progreso, ser más consciente de los procesos que realiza y de cómo puede modificarlos y reflexionar sobre la calidad de sus producciones. Algunas aportaciones recientes (Bennett et al., 2017; Cubero et al., 2018; Broadbent et al., 2020) constituyen referentes valiosos para promover este cambio, pero quizá el discurso teórico va por delante de las prácticas que se están realizando en la actualidad. Por este motivo el 
objetivo de este trabajo es analizar la contribución de las tecnologías digitales en los procesos de feedback autorregulador en la educación superior.

\section{ANTECEDENTES}

La evaluación es un elemento clave para lograr un aprendizaje efectivo, puesto que orienta el aprendizaje. Es el denominado "efecto retroceso" que Biggs y Tang (2003) han documentado para aludir al impacto que la evaluación posee en tanto que los estudiantes plantean una lógica inversa a la del profesor. Mientras que este parte de los objetivos, diseña actividades y finalmente planifica la evaluación, los estudiantes parten de la evaluación, luego enfrentan las tareas que van a tener que realizar y finalmente, los resultados/objetivos a los que se llega.

En los últimos años numerosos autores han documentado la importancia de la evaluación en los procesos de aprendizaje en educación superior. Se ha pasado de focalizar la mirada en la finalidad acreditativa de este proceso (evaluación del aprendizaje) a enfatizar la dimensión formativa de los procesos de evaluación (evaluación para el aprendizaje, recogiendo toda la literatura relativa al AfL, Assessment for Learning) (Stiggins, 2005).

Esta evaluación formativa tiene que inscribirse en el marco de un proceso de evaluación continua para que las valoraciones acerca de la calidad de las respuestas del estudiante (actuaciones, ejercicios o trabajos) puedan ser utilizadas (Black y Wiliam, 1998) durante el proceso para mejorar no solo la tarea final sino el propio proceso de aprendizaje. Para ello ha de proveerse un feedback. Al principio se consideraba que el modo en que el docente estructuraba el feedback era el mejor modo de evitar que los estudiantes aprendiesen por ensayo y error. También se consideraba y continúa siendo muy relevante reflexionar acerca de la estructuración, temporización, contenido y soporte del feedback en cada contexto. Es lo que Black y Wiliam (1998) denominaron "constelaciones" haciendo referencia a todas las posibles decisiones acerca del feedback en virtud de las respuestas que se den a quién, cómo, cuándo, con qué y para qué lo provee. Sin embargo, con el tiempo, este proceso de orientación por parte exclusivamente del profesor ha sido superado por visiones en los que el estudiante tiene un rol más activo. Los modos de concebir el feedback han ido variando y la responsabilidad del mismo ha ido basculando hacia un mayor protagonismo de los estudiantes. 


\section{Tabla 1}

Modos de concebir la retroalimentación o feedback

\begin{tabular}{|c|c|c|}
\hline $\begin{array}{l}\text { Concepciones de } \\
\text { retroalimentación }\end{array}$ & Definición de feedback & $\begin{array}{l}\text { Responsabilidad por parte del } \\
\text { docente y del estudiante. }\end{array}$ \\
\hline $\begin{array}{l}\text { Feedback como } \\
\text { información }\end{array}$ & $\begin{array}{l}\text { El feedback es la información } \\
\text { sobre el rendimiento o la } \\
\text { comprensión de una tarea que } \\
\text { recibe el estudiante (Hattie y } \\
\text { Timperley, 2007). }\end{array}$ & $\begin{array}{l}\text { Es responsabilidad básicamente de } \\
\text { los docentes y ha de ayudar a cubrir } \\
\text { la diferencia entre la ejecución que } \\
\text { realizan los estudiantes y la } \\
\text { ejecución ideal. }\end{array}$ \\
\hline $\begin{array}{l}\text { Feedback como } \\
\text { interacción }\end{array}$ & $\begin{array}{l}\text { El feedback es el diálogo sobre } \\
\text { una tarea para favorecer el } \\
\text { aprendizaje tanto en } \\
\text { situaciones formales como } \\
\text { informales (Carless, 2016). }\end{array}$ & $\begin{array}{l}\text { Constituye una responsabilidad } \\
\text { compartida. } \\
\text { Se trata de dialogar para profundizar } \\
\text { en el sentido de una tarea bien } \\
\text { realizada. }\end{array}$ \\
\hline $\begin{array}{l}\text { Feedback como } \\
\text { acción }\end{array}$ & $\begin{array}{l}\text { El feedback es el conjunto de } \\
\text { acciones que realizan los } \\
\text { estudiantes para dar sentido a } \\
\text { los comentarios recibidos y } \\
\text { emplearlos para mejorar (Boud } \\
\text { y Molloy, 2013; Carless y } \\
\text { Boud, 2018). }\end{array}$ & $\begin{array}{l}\text { Es una responsabilidad del } \\
\text { estudiante. Tras recibir información } \\
\text { sobre su proceso de trabajo o sobre } \\
\text { el trabajo entregado, ha de } \\
\text { involucrarse activamente con el fin } \\
\text { de aplicar los comentarios recibidos } \\
\text { y las reflexiones realizadas a futuros } \\
\text { trabajos. }\end{array}$ \\
\hline
\end{tabular}

Fuente: Cano, Pons y Lluch (2020, p. 5).

Así pues, la evaluación formativa ha de ser necesariamente participada por el estudiante. Boud ha indicado reiteradamente la necesidad de que el estudiante sea el responsable de su propio aprendizaje y de su propia evaluación y demuestre capacidad para juzgar la calidad de su propio trabajo y el trabajo de los otros. Es lo que en principio denominó evaluación sostenible (Boud, 2000) y que últimamente se ha venido denominado juicio evaluativo (Panadero et al., 2018; Tai et al., 2018), entendido como:

La capacidad de tomar decisiones sobre la calidad del propio trabajo y del de los demás e indican que incluye tanto comprender en qué consiste la calidad de una tarea o proceso (conociendo muy bien los criterios y/o procedimientos a desarrollar), como aplicar esta comprensión en la valoración de una tarea, ya sea la propia o de otra persona. (Tai et al., 2018, p. 472)

El juicio evaluativo, entendido de este modo, puede contribuir al aprendizaje autorregulado (control que el sujeto realiza sobre sus pensamientos, acciones, emociones y motivación, a través de estrategias personales) y al desarrollo de la competencia de aprender a aprender. 
Como ya ha sido indicado (Lluch et al., 2020), a partir de las aportaciones de Zimmerman (1986), Pintrich (2000) y Boekaerts y Corno (2005), los procesos de autorregulación poseen una triple dimensión: cognitiva, metacognitiva y emocional.

Las fases cíclicas del aprendizaje autorregulado de Zimmerman (1986) poseen claros paralelismos con las fases del feedback. En la fase de planificación se procede a un análisis de la tarea, se establecen metas, se procede a una planificación estratégica para optimizar el propio rendimiento durante el aprendizaje, seleccionando las estrategias de aprendizaje. En la fase de realización se desarrollan los procesos de aprendizaje en paralelo a la supervisión de la consecución de los objetivos mediante estrategias de autocontrol (que permiten centrarse en la tarea y optimizar el esfuerzo, explicitando los pasos a emprender) y estrategias de auto-observación mediante las cuales el estudiante revisa su propio rendimiento. En la tercera fase, la de autorreflexión se producen juicios escolares con los que se valora el propio rendimiento y se realizan atribuciones causales, y auto-reacciones que promueven las creencias positivas del alumno acerca de sí mismo, lo que lleva a incrementar su percepción de autoeficacia.

El papel de la evaluación en los procesos de autorregulación ha sido sobradamente analizado (Boud, 2000; Tai et al. 2018). La participación en los procesos de evaluación fomenta el desarrollo del juicio evaluativo y la autorregulación (Panadero et al., 2019). Sin embargo, ello no se da sin una planificación explícita. Por ello, es necesario secuenciar y apoyar el proceso de aprendizaje. La evaluación entre iguales ha sido señalada como un estadio intermedio entre la evaluación del docente y la autoevaluación que permite promover el desarrollo del juicio evaluativo y la autorregulación (Tai et al., 2018). Ayuda al estudiante a apropiarse y aplicar los criterios de evaluación y a analizar de qué manera estos han sido considerados en la resolución de la tarea del igual, lo que redunda tanto en la mejora de la propia tarea como del proceso de aprendizaje. Ello ha sido referido por Sanmartí (2010), quien recuerda la necesidad de: (a) Identificar, reflexionar y compartir los objetivos de la actividad; (b) Anticipar y planificar la acción para realizar la tarea y (c) Compartir los criterios de evaluación. La cuestión es si puede la tecnología aportar un valor añadido a los procesos de feedback que permitan tanto el feed-up (representarse la tarea y saber hacia dónde se va), como el feed-back (monitorear el proceso) y, finalmente el feed-forward (cómo transferir estos aprendizajes a futuras tareas, asignaturas o actividades profesionales). Ahí reside el reto de la tecnología: en soportar y promover este tipo de procesos, a partir de un rol activo del estudiante, más que en contribuir al almacenamiento y gestión de datos.

Todos estos procesos pueden recibir el soporte de la tecnología. Sin embargo, Bhagat y Spector (2017) citan a Bennett y Gitomer (2009), quienes enumeraron algunos de los beneficios importantes del uso de la tecnología, señalando que ésta es: (a) más informativa, en tanto que puede ayudar a rastrear el registro completo del proceso de resolución de problemas adaptado por el alumno; (b) más eficiente, 
puesto que ahorra tiempo para puntuar y permite hacerlo sin errores; y (c) más rentable, en el sentido de que ahorra los gastos del proceso de calificación humana.

\section{Tabla 2}

Procesos en los que la tecnología contribuye a la retroalimentación

\begin{tabular}{|c|c|c|c|c|}
\hline \multirow{2}{*}{ TEF System } & \multirow{2}{*}{ Reported by } & \multicolumn{3}{|c|}{ Technology enables feedback by } \\
\hline & & Acquiring & Transforming & Conveying \\
\hline $\begin{array}{l}\text { Websites that } \\
\text { Host Feedback }\end{array}$ & Harrison et.al.(2013) & & & $v$ \\
\hline Audio Feedback & $\begin{array}{l}\text { Cann (2014); Henderson \& Phillips } \\
\text { (2014); Hennessy \& Forrester } \\
\text { (2013); McCarthy (2015) }\end{array}$ & $\checkmark$ & & $\checkmark$ \\
\hline $\begin{array}{l}\text { Video \& } \\
\text { Screencast } \\
\text { Feedback }\end{array}$ & $\begin{array}{l}\text { Barry (2012); Crook et.al. (2012); } \\
\text { Henderson \& Phillips (2014); } \\
\text { Henderson \& Phillips (2015); } \\
\text { Marriott \& Teoh (2012); McCarthy } \\
\text { (2015); West \& Turner (2015); } \\
\text { Yuan \& Kim (2015); Phillips, } \\
\text { Henderson \& Ryan (2016) }\end{array}$ & $\checkmark$ & & $\checkmark$ \\
\hline $\begin{array}{l}\text { Discussion } \\
\text { Forums }\end{array}$ & $\begin{array}{l}\text { Coll et.al. (2013); Shroff \& Deneen } \\
\text { (2011); Huang \& Hung (2013) }\end{array}$ & $\checkmark$ & & $\checkmark$ \\
\hline $\begin{array}{l}\text { Messaging } \\
\text { Systems \& LMS }\end{array}$ & $\begin{array}{l}\text { Horvadas et.al. (2013); Lai \& } \\
\text { Hwang (2015); Burrows \& Shortis } \\
\text { (2011) }\end{array}$ & $\checkmark$ & & $\checkmark$ \\
\hline $\begin{array}{l}\text { Adaptive Grade } \\
\text { Release }\end{array}$ & $\begin{array}{l}\text { Hepplestone et.al. (2011); Irwin } \\
\text { et.al. (2012); Parkin et.al. (2011) }\end{array}$ & $\checkmark$ & & $\checkmark$ \\
\hline $\begin{array}{l}\text { e-Learning } \\
\text { Applications }\end{array}$ & $\begin{array}{l}\text { Shute \& Towle (2003); Van der } \\
\text { Kleij et.al.(2015); Timmers } \\
\text { et.al.(2013) }\end{array}$ & $\checkmark$ & $\checkmark$ & $\boldsymbol{v}$ \\
\hline $\begin{array}{l}\text { Automated } \\
\text { Marking Systems }\end{array}$ & $\begin{array}{l}\text { Jordan (2011); Jordan (2012); } \\
\text { Jordan (2013); Jordan \& Mitchell } \\
\text { (2009); Dikli (2006); Warschauer \& } \\
\text { Ware (2006); Chowdorow et.al. } \\
\text { (2010); }\end{array}$ & $\checkmark$ & $\checkmark$ & $\checkmark$ \\
\hline $\begin{array}{l}\text { Intelligent } \\
\text { Tutoring Systems }\end{array}$ & Narciss (2014) & $\checkmark$ & $\checkmark$ & $\checkmark$ \\
\hline Computer Games & $\begin{array}{l}\text { Shute (2011); Shute \& Ke (2012); } \\
\text { Nino \& Evans (2015) }\end{array}$ & $\checkmark$ & $\checkmark$ & $\checkmark$ \\
\hline
\end{tabular}

Fuente: Deenen y Munshi (2018, p. 115)

Deneen y Munshi (2018) recogen las tres principales finalidades de los procesos en los que el feedback es proporcionado con tecnologías digitales: (1) Recoger y almacenar información del estudiante; (2) Transformar esa información en un mensaje de feedback y (3) Comunicar el feedback a los estudiantes, intentando aumentar su motivación. Pese a la utilidad de la tecnología, parecería necesario superar estas finalidades y añadir una cuarta, vinculada a la contribución de 
la tecnología al aprendizaje autorregulado en la línea que Benett et al. (2017) o Broadbent et al. (2020) sugieren y que da lugar al estudio que se presenta con el objetivo de identificar si las propuestas tecnológicas existentes responden a un uso tradicional del feedback o se emplean para apoyar las diversas fases de planificación, realización y autorreflexión del aprendizaje autorregulado.

\section{METODOLOGÍA}

Se ha llevado a cabo una revisión sistemática de la literatura, entendida como un tipo de estudio que ayuda a identificar, evaluar e interpretar la investigación disponible relacionada con un área temática (Kitchenham y Charters, 2007). Para analizar los artículos de manera rigurosa, se estableció un protocolo de análisis con las siguientes fases: 1) Preguntas de investigación; 2) Proceso de búsqueda; 3) Criterios de inclusión y exclusión; 4) Proceso de selección y extracción de datos; 5) Síntesis de datos (Ramírez y Lugo, 2020).

Fase 1: Preguntas de investigación. Con el objetivo de analizar el papel de la tecnología para el apoyo del aprendizaje autorregulado se plantearon preguntas de investigación para localizar los artículos relativos a los hallazgos del papel de las tecnologías digitales en los procesos de feedback.

Fase 2: Proceso de búsqueda. El protocolo de búsqueda de los artículos integró los procesos electrónicos en las bases de datos Scopus y Web of Science (WoS), delimitando las palabras clave (feedback, feedbak literacy, formative assessment), el idioma (inglés), el período temporal (2015-2020) y el tipo de documento (artículo). El resultado fue de 804 artículos, de los cuales se eliminaron los artículos repetidos quedando un total de 621.

Fase 3: Criterios de inclusión y exclusión de los artículos. Se excluyeron:

A. Aquellos que no fuesen de educación superior.

B. Aquellos que fuesen más cercanos a procesos de elaboración, diseño, validación de instrumentos.

C. Aquellos que fuesen de campos de conocimiento específicos (medicina, biología, física, etc.) y que contuviesen un término relativo a dicho campo de conocimiento en el título (exceptuando el campo de educación o enseñanza).

Aquellos que, pese a pertenecer a la selección inicial, no aportaban información relevante al objeto de estudio, como los relativos a temas de mayor amplitud, vinculados a la política educativa, o a temas muy específicos (como un programa de mejora de la sostenibilidad) donde el feedback no era el elemento central.

Con ello se pretendió mantener únicamente los artículos genéricos. Quedaron 346 documentos. Finalmente se seleccionaron únicamente aquellos que incluyesen technology o technologies en el título, arrojando un resultado de 15 documentos, que aparecen en la Tabla 2. 
Fase 4: Proceso de selección y extracción de datos. Se buscó sistematizar información en torno a los siguientes campos: 1) Objetivo; 2) Contexto; 3) Principales aportaciones del marco teórico; 4) Breve descripción de la experiencia y resultados; 5) Uso que se hace de la tecnología para la provisión del feedback; 6) Rol que tiene el estudiante en el empleo de la tecnología y 7) Herramientas tecnológicas que emplea.

Fase 5: Síntesis de datos. Para sintetizar las contribuciones de cada artículo se procedió a una lectura atenta y a una sistematización de las principales aportaciones en torno a los 7 campos determinados dispuestos en columnas en un documento Excel on line.

\section{Figura 1}

Diagrama de flujo del proceso de selección de textos para la revisión sistemática

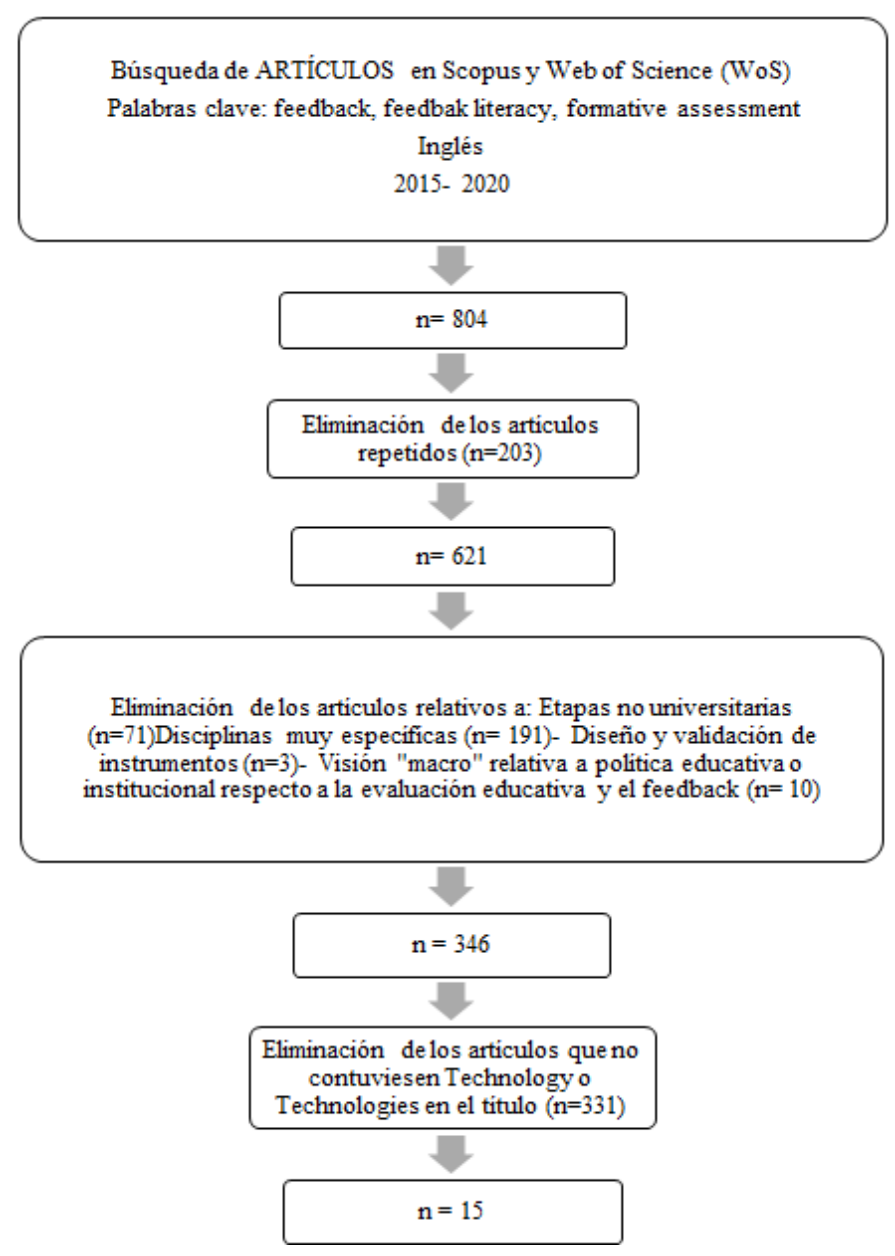


El hecho de iniciar la búsqueda sin incluir explícitamente el término tecnología obedeció al enfoque del estudio, cuyo interés no estaba en analizar instrumentos o el empleo instrumental de la tecnología sino en los beneficios que los procesos feedback participados por el estudiante pueden generar sobre el aprendizaje autorregulado. De ahí que se iniciase la búsqueda con términos exclusivamente vinculados a la alfabetización acerca del feedback y la evaluación formativa y sólo en la fase 4 se seleccionasen los vinculados a la tecnología. Este procedimiento generó un sesgo respecto a los documentos finalmente analizados que se asume como limitación del presente estudio.

\section{RESULTADOS}

Tal y como se muestra en la Tabla 3 , hemos agrupado los trabajos seleccionados en dos grupos: los que presentan una revisión sistemática sobre el tema o realizan una revisión teórica y los que presentan propuestas concretas del uso de la tecnología para la mejora del feedback.

\section{Tabla 3}

Estudios sobre uso de la tecnología en procesos de feedback

\begin{tabular}{|c|c|}
\hline Descripción & Documentos \\
\hline $\begin{array}{l}\text { Revisiones generales y sistemáticas sobre el } \\
\text { papel de la tecnología en procesos de } \\
\text { feedback para fomentar la autorregulación }\end{array}$ & $\begin{array}{l}\text { (García-Jiménez, 2015), (Spector, Ifenthaler, } \\
\text { Samspon, Yang, Mukama, Warusavitarana, } \\
\text { Lokuge, Eichhorn, Fluck, Huang, Bridges, } \\
\text { Lu, Ren, Gui, Deneen, San Diego y Gibson, } \\
\text { 2016), (Yuan y Kim, 2016), (Bhagat y } \\
\text { Spector 2017), (Raposo y Cebrian, 2019), } \\
\text { (Robertson, Humphrey y Steele 2019) }\end{array}$ \\
\hline
\end{tabular}

Propuestas tecnológicas para mejorar el (García-López y García-Mazarío, 2016), feedback basados en cuestionarios de (Robertson, Humphrey y Steele, 2019) autoevaluación

Propuestas tecnológicas basadas en el uso de rúbricas y ensayos

(Yuan y Kim, 2016), (Koskinen, Lämsä, Maunuksela, Hämäläinen, y Viriri,2018), Santamaría, Hernández, Sánchez, Luzón y Jorge, 2018)

\begin{tabular}{l}
$\begin{array}{l}\text { Analíticas de aprendizaje y tutores } \\
\text { inteligentes }\end{array}$ \\
$\begin{array}{l}\text { (Kim, Zouaq y Kim, 2016), (Harindranathan } \\
\text { y Folkestad, 2019), (Said, Aravind, } \\
\text { Ferdinand-James y Umachandran, 2019) }\end{array}$ \\
\hline
\end{tabular}

Fuente: Elaboración propia 
Seis de los trabajos seleccionados hacen una extensa revisión y discusión de la literatura sobre las características más apropiadas que deben tener las herramientas tecnológicas para mejorar los procesos de evaluación. García-Jiménez (2015) realiza un trabajo muy completo en el que se presenta una revisión sobre la evaluación centrada en la información que se proporciona a los estudiantes universitarios a partir del análisis de sus resultados de aprendizaje y la relación entre el modo en que las tecnologías digitales se pueden usar para facilitar que dicha información se utilice para mejorar los aprendizajes. El trabajo contempla diferentes formas de participación de los estudiantes en la evaluación (coevaluación, evaluación entre iguales y autoevaluación) y sus consecuencias en el análisis y aprovechamiento de los resultados de la evaluación. En cuanto a los beneficios de las tecnologías en los procesos de evaluación se indica que aportan inmediatez y mayor precisión de la información que se proporciona. Respecto a los usos de las tecnologías, señala que "se utilizan para el registro y almacenamiento del desempeño y posterior análisis y revisión, para presentar modelos de trabajos bien hechos o para enviar información u orientaciones sobre una tarea" (García-Jiménez, 2015, p. 18).

Spector et al. (2016) analizan los problemas actuales de la evaluación formativa y consideran que las tecnologías disponibles pueden ayudar a desarrollar nuevas prácticas relacionadas con el pensamiento crítico y la resolución de problemas complejos. Consideran que las tendencias y retos más importantes en las evaluaciones formativas mediadas por la tecnología digital son las siguientes: (a) el andamiaje, que es una parte importante del feedback formativo que puede ser apoyado por tecnologías basadas en tutores inteligentes, agentes pedagógicos; (b) las herramientas para apoyar el aprendizaje basado en problemas como, por ejemplo, los portafolios digitales y (c) la evaluación adaptativa que permite ofrecer feedback personalizado entre estudiantes.

En una línea similar, Robertson et al. (2019) a partir de una revisión bibliográfica crean una lista de las características principales que deben proporcionar las herramientas tecnológicas para apoyar la evaluación y el feedback, entre las que destacan: (a) la inmediatez; (b) la posibilidad de realizar comentarios elaborados del profesorado; (c) la posibilidad de incluir comentarios personalizados de los estudiantes; (d) la posibilidad de reutilización; (e) la accesibilidad; (f) el diseño de la interfaz; (g) la interacción y (h) el coste. Con todo ello generan un instrumento de revisión de objetos de aprendizaje (LORI) compuesto de 9 criterios de cumplimiento de los estándares: reutilización, accesibilidad, usabilidad de la interacción, diseño de la presentación, motivación, retroalimentación y adaptación, alineación de los objetivos de aprendizaje y calidad del contenido.

Yuan y Kim (2016) también realizan una revisión sistemática y concluyen que la eficacia no depende de la tecnología sino del diseño del feedback, que es lo que maximiza las posibilidades de la tecnología en aras a un rol más activo del estudiante. Respecto a la calidad del feedback, esta depende tanto del contenido (que sea específico y que informe acerca de cómo hacer mejoras); como del timming (que 
sea cercano) y del diálogo que puede generar (los estudiantes tienen que comprender la retroalimentación para usarla). La tecnología debería contribuir a todo ello. Por otra parte, respecto al rol, cuando los estudiantes brindan retroalimentación a sus compañeros, aumenta su participación en las tareas de aprendizaje y desarrollan una mejor comprensión de los criterios de evaluación, pudiendo diagnosticar problemas con su propio trabajo e idear propuestas para mejorar. En este sentido, Yuan y Kim (2016) parten del supuesto de que la retroalimentación es ineficaz a menos que los estudiantes actúen en consecuencia. Para tomar decisiones más informadas es conveniente que les llegue información de diversas fuentes y que puedan establecerse canales de comunicación. Sin embargo, las oportunidades para la retroalimentación múltiple y la comunicación adicional sobre la retroalimentación son limitadas en los cursos en línea debido a las dificultades para interactuar entre instructores y estudiantes, así como entre estudiantes. Por ello, proporcionar comentarios efectivos es un desafío en los entornos en línea porque los estudiantes pueden ignorar fácilmente los mensajes de los instructores que se encuentran entre una gran cantidad de otros comentarios y materiales en línea. Asimismo, también es un desafío para los instructores alentar a los estudiantes a proporcionar retroalimentación de sus compañeros debido a las dificultades de interacción entre los estudiantes. Por ello consideran que las tecnologías se pueden usar para mejorar la efectividad de la retroalimentación en los cursos en línea.

Raposo y Cebrián (2019) también señalan la importancia de las tecnologías digitales para mejorar los procesos de evaluación diagnóstica, sumativa y formativa, así como para personalizar la enseñanza, comunicar y reflexionar sobre lo aprendido. Las tecnologías digitales permiten que la retroalimentación sea más interactiva e instantánea lo que facilita el desarrollo de la formación on-line. En este trabajo se insiste en la necesidad de desarrollar tecnologías que no sólo se focalicen en el feedback del profesorado sino también en la autoevaluación y la evaluación por pares. Entre las múltiples tecnologías que pueden utilizarse se destacan: (a) los portafolios digitales; (b) las rúbricas electrónicas; (c) los sistemas de anotaciones multimedia que permiten realizar anotaciones sobre videos e imágenes y (d) el uso de blockchain para generar itinerarios de aprendizaje individualizados.

Entre los trabajos que presentan propuestas tecnológicas para mejorar el feedback hay bastante diversidad sobre las tecnologías utilizadas y también sobre la intencionalidad pedagógica. En algunos casos, se focaliza el uso del feedback sobre la comprensión de una tarea. Por ejemplo, Robertson et al. (2019) realizan un estudio con 286 estudiantes de Estados Unidos con el fin de reportar los efectos del uso de Socrative sobre la participación de los estudiantes, las calificaciones, el tiempo transcurrido entre la prueba y el feedback y el tiempo dedicado por el profesorado a la calificación. El uso de esta tecnología reporta beneficios en términos de motivación y participación de los estudiantes. El tiempo también parece ser relevante, tanto en términos de la inmediatez que las herramientas tecnológicas, en este caso Socrative, pueden aportar como en el ahorro de tiempo de calificación por parte del profesorado. 
Sin embargo, el análisis mediante $t$ de Student para muestras independientes no muestra diferencias en la puntuación de los alumnos que usan Socrative. El estudio concluye que la herramienta empleada puede ayudar a los estudiantes a prepararse para la evaluación sumativa, con lo que la noción que hay tras su empleo parece ser instrumental y no constituir un fin en sí mismo que contribuya al desarrollo ni de competencias digitales ni de la competencia de aprender a aprender.

García López y García Mazarío, (2016) analizan la experiencia de combinar en un curso de matemáticas, cuestionarios de autoevaluación en Moodle con la creación de una comunidad de aprendizaje en línea para facilitar la evaluación por pares. Los resultados son positivos, pero señalan que es difícil utilizar diferentes entornos de comunicación si no están bien integrados. El mantenimiento por separado del entorno Moodle y la red social en línea complica el trabajo y obliga a los involucrados a utilizar diferentes espacios de trabajo.

En una línea diferente, Van der Kleij et al. (2015) analizan el uso del videofeedback. Diseñan e implementan un estudio en el que registran conversaciones de feedback en 6 parejas de docente y estudiante. Tras grabar el vídeo, el docente y el estudiante por separado participan en unas entrevistas con "video-stimulated recall (VSR)", desarrollando reflexiones que son registradas. Los participantes paran el video en los puntos que ellos consideran relevantes y los comentan. La finalidad era comprender las interpretaciones del feedback por parte de los docentes y los estudiantes y los aspectos que consideraban importantes y válidos de la conversación. Los docentes y los estudiantes reportan comprensiones diferentes sobre la naturaleza del significado de los componentes del proceso de feedback. Este estudio apunta que el uso de video-feedback puede ayudar a los estudiantes a reflexionar sobre el contenido de las respuestas recibidas identificando qué contenido deben aprender y qué estrategias deben aplicar. Asimismo, el video-feedback puede ayudar a los docentes a reflexionar sobre los procesos de feedback que llevan a la práctica. En concreto el VSR puede tener el potencial de ayudar a los estudiantes a reflexionar sobre el feedback.

Santamaría et al. (2018) realizan una intervención consistente en dar retroalimentación a preguntas abiertas a través de tecnologías semánticas, derivadas del AEA (Evaluación Automática de Ensayos), de manera sostenible basándose en análisis semántico latente (LSA) de preguntas abiertas a partir del G-rubric para apoyar el papel de los tutores on line. El objetivo es automatizar las correcciones de los ensayos para que la fiabilidad tanto entre evaluadores como del mismo evaluador en diversas pruebas aumente. El contexto en el que se realiza la intervención es un curso de la UNED sobre historia económica. En síntesis, la aportación de Santamaría et al. (2018) es relativa a la automatización de la corrección de las preguntas abiertas. La tecnología de feedback formativo de respuestas abiertas, basadas en un análisis semántico latente, permite la participación del estudiante que puede reflexionar sobre el feedback y reelaborar sus respuestas. Con unos ensayos previos y a partir del texto redactado por el profesor se determina un corpus de palabras que emplean 
para evaluar el primer borrador, dar un feedback automatizado y ver si se "corrige" en el segundo borrador. El estudio documenta que la satisfacción de los estudiantes es alta, así como la percepción de mejor preparación para el examen final y mejora de las calificaciones en el $2^{\circ}$ borrador de aquellos que reciben feedback. Por otra parte, los resultados de la prueba parecen mostrar que la interacción con G-Rubric puede mejorar el aprendizaje al brindar retroalimentación detallada.

Yuan y Kim (2016) realizan un estudio en el que proponen a los estudiantes que tengan un rol activo, haciendo uso de tres herramientas: realizando anotaciones multimedia y en colores con Voice Thread; discutiendo en la wiki (en su defecto, foro de Moodle) e intercambiando pantallas capturadas (mediante Jung) con los pares e ilustran los beneficios que la participación y el diálogo aportan.

Koskinen et al. (2018) valoran la aplicación de este modelo que se puso a prueba en el 2016 y 2017 en un curso de física universitaria sobre termodinámica y óptica de 7 semanas de duración. Los cursos tuvieron 72 (2016) y 77 (2017) estudiantes que se dividieron en grupos de cinco alumnos que fueron formados por el profesor. Los resultados de los cursos pilotos en 2016 y 2017 se compararon con los cursos en 2014 y 2015 ya que tenían el mismo contenido, los mismos profesores y un número similar de estudiantes. Los resultados muestran que la aplicación del modelo mejoró la retención y prolongó la actividad estudiantil. La distribución total de puntos fue cualitativamente similar a la evaluación sumativa de años anteriores. El modelo mejoró la retención, la carga de trabajo y la disminución de errores. Además, los comentarios de las sesiones de práctica muestran que el uso de la tecnología mejoraba el aprendizaje autónomo.

Harindranathan y Folkestad (2019) analizan los resultados de un curso de microbiología desarrollado con Canvas y que se estructuró de forma cíclica: se presenta el contenido y tareas a través de un video, durante 9 días los estudiantes han de realizar las actividades asociadas y pueden usar un conjunto de pruebas para valorar su conocimiento. El ciclo se cierra con un examen que hay que realizar antes de pasar a la siguiente tarea. La investigación pretende determinar qué datos relativos a las actividades de los estudiantes resultan más útiles y si hay una mejora de las calificaciones. Con relación a la primera pregunta, las variables que parecen resultar más interesantes para el profesorado y los estudiantes son: el tiempo total utilizado, la actividad cercana a la entrega de tarea, el número de intentos y el tiempo entre sesiones. En cuanto a los resultados de las calificaciones se obtienen claras mejoras entre la conducta productiva dentro del campus y los resultados finales.

Son escasos los trabajos que aportan resultados sobre el feedback como acción. La aportación más relevante es la de Koskinen et al. (2018) que elaboran lo que denominan "the primetime learning model" que consiste en un modelo que reúne los elementos básicos del aprendizaje activo basados en el aprendizaje colaborativo y el aprendizaje mediado por la tecnología. Bajo este enfoque, la tecnología se contempla como soporte que permite que los estudiantes interactúen con sus iguales 
para articular pensamientos y argumentos, para proporcionar diversas visiones y representaciones múltiples y utilizar contextos ricos usando tecnologías multimedia.

Harindranathan y Folkestad (2019) también afirman que las tecnologías digitales tienen un papel muy relevante para apoyar la autorreflexión y la autorregulación. No obstante, creen que hay pocas evidencias sobre qué tipos de diseños son más adecuados para mejorar la autorregulación y critican que, en las universidades presenciales, los campus virtuales se han utilizado de forma masiva para distribuir materiales y no para generar actividades de aprendizaje. Estos autores insisten en la necesidad de utilizar las analíticas para mejorar, al mismo tiempo, el diseño formativo y el proceso de aprendizaje de los estudiantes. Para ello, elaboran un modelo pedagógico que permite analizar constantemente el diseño de las actividades de aprendizaje a través de los datos que se obtienen que, a su vez, sirven para refinar el diseño de las tareas.

A un nivel de mayor complejidad encontramos diversas investigaciones centradas en el desarrollo de tutores inteligentes. En este sentido, Kim et al. (2016) valoran el impacto de un tutor inteligente para ayudar a los estudiantes en el proceso de solución de problemas en el contexto del aprendizaje de la Física. El prototipo diseñado se aplicó a 14 estudiantes de un curso de introducción a la física. Se trata de un curso on-line en el que se presentan problemas a resolver y el sistema proporciona respuesta inmediata (correcto, no correcto, pistas para resolver el problema). El estudiante puede pedir ayuda al sistema sin esperar al final para elaborar la respuesta. Los resultados obtenidos muestran que los estudiantes piden ayuda al sistema desde el inicio de la actividad, pero, a medida que avanzan, requieren de menos intervención del sistema. Los estudiantes se centran en la mejora de sus respuestas y no se comparan con el resto. En general, el uso del tutor inteligente muestra una progresión más rápida que en cursos anteriores.

Said et al. (2019) también analizan el uso de un tutor inteligente. Estos autores proponen realizar un cambio de paradigma de la evaluación tradicional (centrada en el profesor) a la evaluación mejorada por la tecnología (centrada en el alumno). Se basan en la teoría del aprendizaje situado que aborda las necesidades e inquietudes de los estudiantes a partir de experiencias de aprendizaje próximas y contextualizadas. Se realizó un estudio de caso con una muestra intencionada de 14 estudiantes de física. Recibieron el apoyo de un sistema de tutoría inteligente para monitorear su aprendizaje con retroalimentación correctiva. Los resultados de las evaluaciones acumulativas fueron mejores que en años anteriores y, además, se concluye que los estudiantes se centran más en los aspectos positivos y se reduce la ansiedad y la fobia a la comparación. 


\section{CONCLUSIONES}

Las publicaciones analizadas presentan desarrollos tecnológicos muy variados que responden claramente a los tipos de feedback referenciados al inicio del artículo (Cano et al., 2020). En la mayoría de los casos, la tecnología utilizada proporciona información sobre el rendimiento o la comprensión de una tarea (Harindranathan y Folkestad, 2019). Las aportaciones que entienden el feedback como interacción son muy variadas en cuanto a propuestas y enfoques. García López y García Mazarío (2016), Yuan y Kim (2016) y Koshinen et al. (2018) se encuentran en esta línea de focalizarse en la interacción. Sin embargo, son escasos los trabajos que aportan resultados sobre el feedback como acción.

Tal y como mencionan Spector et al. (2016) actualmente casi todos los entornos de aprendizaje involucran y dependen de las tecnologías digitales. El propósito de la evaluación formativa no ha cambiado debido a las nuevas tecnologías. Más bien, la importancia de la evaluación formativa ha aumentado gracias a las nuevas tecnologías y las demandas de aprendizaje del siglo XXI. En este sentido, Spector et al. (2016) consideran que las tecnologías disponibles pueden ayudar a desarrollar nuevas prácticas relacionadas con el pensamiento crítico y la resolución de problemas complejos. Por ello, las evaluaciones acumulativas, como las calificaciones, las pruebas estandarizadas y las clasificaciones comparativas no son suficientes para la evaluación formativa que debe proporcionar retroalimentación individualizada y constructiva durante la formación. El feedback del profesor al estudiante no debería ser la única forma de proporcionar retroalimentación para mejorar los aprendizajes. Tanto la autoevaluación como la evaluación por pares fomentan y fortalecen las habilidades de metacognición y autorregulación que son importantes habilidades de aprendizaje. Sin embargo, las tecnologías no suelen aportar un valor añadido para el desarrollo de dichos procesos.

Tras el análisis de la literatura, se puede afirmar que el hecho de que las tecnologías digitales pueden contribuir a la mejora de la participación de los estudiantes queda sobradamente documentado, tal y como también han recogido Pinto y Leite (2020). Sin embargo, esta participación a menudo se enmarca en un paradigma de enseñanza-aprendizaje tradicional, de modo que se reduce a responder cuestionarios con clickers para mantener la atención y/o lograr mejorar la motivación en entornos de docencia presencial o en línea organizada básicamente en torno a contenidos.

Siguiendo el modelo SAMR (Sustitución, Aumento, Modificación, Redefinición) (Puentedura, 2008) sobre la integración de las TIC en el diseño de actividades, se evidencia que, en la mayor parte de los trabajos analizados, la tecnología se aplica como un elemento sustitutorio de otro preexistente pero no hay una modificación o redefinición. Se producen algunas mejoras funcionales (por ejemplo, mayor velocidad en el feedback). Sin embargo, no se desarrollan redefiniciones significativas de las actividades ni se crean nuevos ambientes de aprendizaje. 
Los análisis sobre el cambio de rol o el cambio de procesos cognitivos y metacognitivos que la tecnología puede promover en el estudiante son, sin embargo, escasos. El valor de las tecnologías digitales en el futuro más que radicar en dar soporte a procesos de aprendizaje desde el punto de vista instrumental o como herramientas al servicio del mismo quizás deberá progresar hacia formas de transformar el modo de aprender.

El reto de empoderar a los estudiantes de sus propios procesos de aprendizaje, para que desarrollen el juicio evaluativo (a través de experiencias de evaluación entre iguales o de autoevaluación, entre otras) sigue siendo una tarea poco explorada. El valor añadido que la tecnología puede reportar a dicho juicio evaluativo, entendido como un elemento que fortalece los procesos de autorregulación del aprendizaje, sea en la fase de planificación, de monitoreo o de autoevaluación, está aún por determinar.

Finalmente, hay que señalar que, aunque los resultados presentados están limitados por el número de trabajos analizados pueden constituir una base para seguir avanzando e investigando sobre la integración de la tecnología para fomentar la autorregulación.

\section{AGRADECIMIENTO}

La contribución está financiada por la Agencia Estatal de Investigación (AEI) Referencia del proyecto/ AEI/ PID2019-104285GB-Ioo.

\section{REFERENCIAS}

Barberà, E. (2016). Aportaciones de la tecnología a la e-Evaluación. Revista De Educación a Distancia (RED), 50. https://doi.org/10.6018/red/50/4

Bennett, S., Dawson, P., Bearman, M., Molloy, E., y Boud, D. (2017). How technology shapes assessment design: Findings from a study of university teachers. British Journal of Educational Technology, 48(2), 672-682. https://doi. org/10.1111/bjet.12439

Bhagat K. K., y Spector J. M. (2017). Formative assessment in complex problemsolving domains: The emerging role of assessment technologies. Educational Technology and Society, 2o(4), 312-317. https://www.scopus.com/inward/record. uri?eid=2-s2.0-85032206740ypartnerID
$=40 y m d 5=8 d_{0 b 948 a 16 a 24} \mathrm{cfo} 136186304$ ob44614

Biggs, J. B., y Tang, C. (2003). Teaching for quality learning at university: What the student does. Buckingham: The Society for Research into Higher Education and Open University Press.

Black, P., y Wiliam, D. (1998). Assessment and classroom learning. Assessment in Education, 5(1), 7-74. https://doi. org/10.1080/0969595980050102

Boekaerts, M., y Corno, L. (2005). Selfregulation in the classroom: A perspective on assessment and intervention. Applied Psychology: An International Review, 54(2), 199-231. https://doi.org/10.1111/ j.1464-0597.2005.00205.x

Boud, D. (2000). Sustainable assessment: rethinking assessment for the learning 
society. Studies in Continuing Education, 22, 2, 151-167. https://doi. org $/ 10.1080 / 713695728$

Boud, D., y Molloy, E. (2013). Rethinking models of feedback for learning: The challenge of design. Assessment and Evaluation in Higher Education, 38(6), 698-712. https://doi.org/10.1080/02602 938.2012.691462

Broadbent, J., Panadero, E., Lodge, J. M., y de Barba, P. (2020). Technologies to enhance self-regulated learning in online and computer mediated learning environments. En M. J. Bishop, J. Elen, E. Boling y V. Svihla (Eds.), Handbook of Research in Educational Communications and Technology. Springer. https://doi. org/10.1007/978-3-030-36119-8 3

Cano, E., Pons, L., y Lluch, L. (2020). Guía Feedback en la educación superior. Barcelona: UB. http://diposit.ub.edu/ dspace/bitstream/2445/158466/1/Guia feedback Educacio Superior Cano Pons Lluch.pdf

Carless, D. (2016). Feedback as Dialogue. Encyclopedia of Educational Philosophy and Theory. Springer Science + Business Media. https://doi.org/10.1007/978-981287-532-7 389-1

Carless, D., y Boud, D (2018). The development of student feedback literacy: enabling uptake of feedback. Assessment and Evaluation in Higher Education. https://doi.org/10.1080/02602938.2018 .1463354

Cubero, J., Ibarra, M. S., y Rodríguez G. (2018). Development and use of mobile technologies that foster students' evaluative judgement: a designbased research. Conference paper. Technological Ecosystems for Enhancing Multiculturality, TEMM'18, Salamanca, Spain, (pp. 151-156). https://doi. org/10.1145/3284179.3284207

Deneen, C., y Munshi, C. (2018). Technologyenabled feedback: It's time for a critical review of research and practice. En M.
Campbell, J. Willems, C. Adachi, D. Blake, I. Doherty, S. Krishnan, S. Macfarlane, L. Ngo, M. O’Donnell, S. Palmer, L. Riddell, I. Story, H. Suri y J. Tai (Eds.), Open Oceans: Learning without borders. Proceedings ASCILITE 2018 Geelong, pp. 113-120.

EACEA (2019). Eurydice Brief. Digital Education at School in Europe. Luxemburg: Eurydice.

EDUCAUSE (2020). Horizon Report. Teaching and Learning Edition. https:// tinyurl.com/yyqerf6b

García-Jiménez, E. (2015). La evaluación del aprendizaje: de la retroalimentación a la autorregulación. El papel de las tecnologías. Revista Electrónica de Investigación y Evaluación Educativa, RELIEVE, 21(2), art. M2. https://doi. org/10.7203/relieve.21.2.7546

García López, A., y García Mazarío, F. (2016). The use of technology in a model of formative assessment. JOTSE: Journal of Technology and Science Education, 6(2), 91-103. https://doi.org/10.3926/ jotse. 190

Gikandi J. W., y Morrow J. W. (2014). Designing and implementing peer formative feedback within online learning environments. Technology, Pedagogy and Education, 25(2), 153-170. https://doi. org/10.1080/1475939X.2015.1058853

Gros, B., y Suárez, C. (Coord). (2016). Pedagogía Red. Una educación para tiempos de internet. Ediciones Octaedro.

Harindranathan, P., y Folkestad, J. (2019). Learning analytics to inform the learning design: Supporting instructor's inquiry into student learning in unsupervised technology enhanced platforms. Online Learning, 23(3), 34-55. https://doi. org/10.24059/olj.v23i3.2057

Hattie, J., y Timperley, H. (2007). The power of Feedback. Review of Educational Research, 77(1), 81-112. https://doi. org/10.3102/003465430298487 
Kim, M. K., Zouaq, A., y Kim S. M. (2016). Automatic detection of expert models: The exploration of expert modeling methods applicable to technology-based assessment and instruction. Computers y Education. https://doi.org/10.1016/j. compedu.2016.05.007

Kitchenham, B., y Charters, S. (2007). Guidelines for performing systematic literature reviews in software engineering. Keele University y University of Durham. https://bit.ly/2LmHwbj

Koskinen, P., Lämsä, J., Maunuksela, J., Hämäläinen, R., y Viiri, J. (2018). Primetime learning: collaborative and technology-enhanced studying with genuine teacher presence. International Journal of STEM Education, 5(1), 20. https://doi.org/10.1186/s40594-0180113-8

Lluch, L., Pons, L., y Cano, E. (2020). La evaluación entre iguales para contribuir al desarrollo de la competencia de aprender a aprender en el grado de maestro de educación primaria. En R. Roig-Vila (Coord.), Redes de Investigación en Docencia Universitaria (pp. 273-284). Alicante: ICE de la Universidad de Alicante.

Panadero, E., Broadbent, J., Boud, D., y Lodge, J. M. (2019). Using formative assessment to influence self- and coregulated learning: The role of evaluative judgement. European Journal of Psychology of Education. https://doi. org/10.1007/s10212-018-0407-8

Pinto, M., y Leite, C. (2020). Digital technologies in support of students learning in Higher Education: literature review. Digital Education Review, 37, 343-360. https://doi.org/10.1344/ der.2020.37.343-360

Pintrich, P. R. (2000). The Role of Goal Orientation in Self-Regulated Learning. Handbook of Self-Regulation, 451502. $\quad$ https://doi.org/10.1016/B978012109890-2/50043-3
Puentedura, R. (2008). Models for enhancing technology integration. http://formacion. intef.es/pluginfile.php/43578/mod imscp/content/1/modelo samr.html

Ramírez; M. S., y Lugo, J. (2020). Revisión sistemática de métodos mixtos en el marco de la innovación educativa. Comunicar, 65(28), 9-20. https://doi.org/10.3916/ C65-2020-01

Raposo, M., y Cebrián, M. (2019). Technology to improve the assessment of learning. Digital Education Review, 35, 1-13.

Robertson S. N., Humphrey S. M., y Steele J. P. (2019). Using technology tools for formative assessments. Journal of Educators, 16(2). https://doi. org/10.9743/JEO.2019.16.2.11

Rodríguez, G., e Ibarra, M. S. (Eds.) (2011). e-Evaluación orientada al e-Aprendizaje estratégico. Narcea,

Said, M. M. T, Aravind, V. R., FerdinandJames, D., y Umachandran, K. (2019). Dissecting assessment: A paradigm shift towards technology-enhanced assessments. World Journal on Educational Technology: Current Issues, 11(2), 24-32.

Sanmartí, N. (2010). Avaluar per aprendre. Generalitat de Catalunya. https://tinyurl. com/y2622dbc

Santamaría, M., Hernández, M., Sánchez, Á., Luzón, J. M., y Jorge, G. (2018). Using Semantic Technologies for Formative Assessment and Scoring in Large Courses and MOOCs. Journal of Interactive Media in Education, 1. https://doi.org/10.5334/ jime.468

Spector, J. M., Ifenthaler, D., Samspon, D., Yang, L., Mukama, E., Warusavitarana, A., Lokuge Dona, K., Eichhorn, K., Fluck, A., Huang, R., Bridges, S., Lu, J., Ren, Y., Gui, X., Deneen, C. C., San Diego, J., y Gibson, D. C. (2016). Technology Enhanced Formative Assessment for 21st Century Learning. Educational Technology y Society, 19(3), 58-71. 
Stiggins, R. (2005). From Formative Assessment to Assessment for Learning: A Path to Success in Standards-Based Schools. The Phi Delta Kappan, 87(4), 324-328. $\quad$ http://www.jstor.org/ stable/20441998

Tai, J., Ajjawi, R., Boud, D., Dawson, P., y Panadero, E. (2018). Developing Evaluative judgment: enabling students to make decisions about the quality of work. Higher Education, 76(3), 467-481. https://doi.org/10.1007/s10734-0170220-3

UNESCO (2020). The digital transformation of education: connecting schools, empowering learners. https://unesdoc. unesco.org/ark:/48223/pfo000374309
Van der Kleij, F., Adie, L., y Cumming, J. (2015). Using video technology to enable student voice in assessment feedback. British Journal of Educational Technology 22(1), 31-41. https:// ddoi/10.1111/bjet.12536

Yuan J., y Kim C. (2016). Effective feedback design using free technologies. Journal of Educational Computing Research, 52(3), 408-434. https://doi. org/10.1177/0735633115571929

Zimmerman, B. J. (1986). Becoming a self-regulated learner: Which are the key subprocesses? Contemporary Educational Psychology, 11(4), 307313. https://doi.org/10.1016/0361476X(86)90027-5

\section{PERFIL ACADÉMICO Y PROFESIONAL DE LAS AUTORAS}

Begoña Gros Salvat. Profesora Catedrática de la Facultad de Educación de la Universidad de Barcelona (España). Directora del Grupo de Investigación de Entornos y Materiales para el aprendizaje. https://orcid.org/0000-0002-5395-7282 E-mail: $\underline{\text { bgros@ub.edu }}$

Elena Cano García. Profesora Titular del Departamento de Didáctica y Organización Educativa de la Facultad de Educación de la Universidad de Barcelona (España). Directora del Grupo de Investigación Consolidado LMI (Learning, Media \& Social Interactions). https://orcid.org/o000-0003-2866-5058

E-mail: ecano@ub.edu

\section{DIRECCIÓN DE LAS AUTORAS}

Campus Mundet, Passeig Vall d'Hebrón, 171. 0835 Barcelona

Fecha de recepción del artículo: 13/11/2020

Fecha de aceptación del artículo: 19/02/2021

Fecha de aprobación para maquetación: 12/03/2021 